\title{
Analysing the Clinical Features of Pneumomediastinum Associated with Diabetic Ketoacidosis in 79 Cases
}

This article was published in the following Dove Press journal: Diabetes, Metabolic Syndrome and Obesity: Targets and Therapy

\author{
Weidong Zhang* \\ jingfang Chen* \\ Xiaoming Wu \\ Luyu Chen \\ Jinxing Wei \\ Mingqiang Xue \\ Qingzheng Liang
}

Department of Thoracic Surgery, Henan Provincial Chest Hospital, Zhengzhou, Henan Province 450003, People's

Republic of China

*These authors contributed equally to this work
Correspondence: Weidong Zhang Room I, Weiwu Road, Zhengzhou City, Henan Province 450003, People's Republic of China

Email zhangweidong037I@I26.com
Objective: To analyse the clinical features of pneumomediastinum associated with DKA (diabetic ketoacidosis) to improve clinicians' understanding of the disease.

Methods: A total of 78 patients with pneumomediastinum associated with DKA were identified in the literature search, and one patient treated in our hospital was included. The clinical features of the 79 patients were retrospectively analysed, and the pathogenesis, clinical symptoms, diagnostic methods, treatment strategies and prognoses were explored.

Results: All cases were confirmed by chest CT (computed tomography), and Kussmaul respiration, severe vomiting, chest pain were common symptoms. The main treatment principle was to correct acidosis and treat the primary disease. The majority of patients recovered within 1-2 weeks, and only 2 patients died.

Conclusion: Pneumomediastinum associated with DKA is a rare disease, and it has a benign course when an early diagnosis is made and aetiological treatment is administered. Keywords: pneumomediastinum, diabetic ketoacidosis, treatment, diagnosis

\section{Introduction}

Pneumomediastinum (PM) is a pathological manifestation of air entering the connective tissue space in the mediastinum pleura and being stored in the mediastinum for various reasons. Most cases of PM are caused by chest trauma, oesophageal perforation, or iatrogenic injury. The incidence of PM is low, approximately $1 / 33,000$, and the proportion of the population with PM between 5 and 34 years old is slightly higher, approximately $1 / 25,000 .^{1-4}$ Pneumomediastinum caused by DKA is rare. The first complete report of this condition was described by Hamman ${ }^{5}$ in 1939, and approximately 78 cases have been reported in the literature to date. This paper reports a 17-year -old female patient who was admitted to our hospital because of pneumomediastinum. After excluding various common causes of pneumomediastinum, we finally diagnosed DKA as the cause of pneumomediastinum. The data of 78 patients with DKA complicated with pneumomediastinum were analysed retrospectively, and the characteristics of diabetic ketoacidosis complicated with pneumomediastinum were summarized. The pathogenesis, clinical symptoms, diagnostic methods and treatment strategies were analysed, and this information can improve clinicians' understanding of the disease.

\section{Case Report}

A 17-year-old female patient with complaints of "chest tightness, shortness of breath for 4 days and blurred consciousness for $5 \mathrm{hrs"} \mathrm{was} \mathrm{admitted} \mathrm{to} \mathrm{our} \mathrm{hospital} \mathrm{in}$ 
July 2017. The patient had symptoms of nausea, vomiting, drinking and polyuria one week prior. At that time, she was given symptomatic treatment for gastroenteritis, and no improvement was observed. The symptoms of chest tightness and shortness of breath appeared 4 days prior and were not considered in the treatment. One day ago, chest tightness and shortness of breath were obviously aggravated. Mediastinal gas was found by a chest CT examination in the local hospital, so she was sent to our hospital through the emergency channel. On the way to the hospital, she exhibited a gradual vagueness of consciousness and a slow response. The patient denied that she had a previous history of diabetes or other diseases, we learned that the patient had a habit of drinking sweet drinks (such as Coca Cola, Sprite), and the patient did not smoke or drink alcohol.

According to the physical examination, her temperature was $36.6^{\circ} \mathrm{C}$, pulse was $130 \mathrm{bpm}$, respiratory rate was 35 per minute, and blood pressure was $108 / 61 \mathrm{mmHg}$. She had poor spirits, lethargy, mild dehydration, and a small amount of crepitation at the entrance to the anterior thoracic neck, her breathing was deep and rapid (Kussmaul breathing), she were smelled the smell of rotten apple, and both lungs were auscultated and exhibited clear sounds without dry or wet sounds. Her heart rate was $130 \mathrm{bpm}$ (normal rhythm), the precordial auscultation heart sound was remote, the friction sound was synchronized with the heart rhythm (Hamman sign, the unique sign of pneumopericardium), and pathological noises were not heard.
According to the assistant examinations, the chest CT showed extensive air in the neck subcutaneously and mediastinum, which had spread along the bronchovascular bundle, and no pneumothorax was found in the bilateral chest (Figure 1). The arterial blood gas analysis results were as follows (oxygen inhalation $3 \mathrm{~L} / \mathrm{min}$ ): $\mathrm{pH}$ of 6.90 (reference range: 7.35 to 7.45 ), partial pressure of oxygen of $144 \mathrm{mmHg}$ (83 108 $\mathrm{mmHg}$ ), partial pressure of carbon dioxide of 10 $\mathrm{mmHg}$ (35 48 $\mathrm{mmHg}$ ), residual alkali could not be detected, and bicarbonate concentration of $9.0 \mathrm{mmol} / \mathrm{L}$. Her white blood cell count was $22.45 \times 10^{9} / \mathrm{L}\left(4-10 \times 10^{9} / \mathrm{L}\right)$, neutrophil level was $79.1 \%(50-70 \%)$, blood glucose concentration was $40.62 \mathrm{mmol} / \mathrm{L}(3.5-6.1 \mathrm{mmol} / \mathrm{L}), \mathrm{HbA1C1}$ level was 3.5\% (4.0-6.0\%), creatinine concentration was $123.6 \mu \mathrm{mol} / \mathrm{L}$ (40-97 $\mu \mathrm{mol} / \mathrm{L})$, urea nitrogen concentration was $7.2 \mathrm{mmol} / \mathrm{L}$ (1.7-8.3 $\mathrm{mmol} / \mathrm{L})$, serum potassium concentration was 3.24 $\mathrm{mmol} / \mathrm{L}(3.5-5.5 \mathrm{mmol} / \mathrm{L})$, serum sodium concentration was $119.0 \mathrm{mmol} / \mathrm{L}$ (135-155 mmol/L), serum chlorine concentration was $89 \mathrm{mmol} / \mathrm{L}(90-110 \mathrm{mmol} / \mathrm{L})$, glutamic pyruvic transaminase concentration was $13.2 \mathrm{U} / \mathrm{L}(0-40 \mathrm{U} / \mathrm{L})$, glutamic oxaloacetic transaminase concentration was $19.9 \mathrm{U} / \mathrm{L}(0-$ $40 \mathrm{U} / \mathrm{L})$, and total protein concentration was $72.4 \mathrm{~g} / \mathrm{L}(60.0$ $85.0 \mathrm{~g} / \mathrm{L})$. Her urine glucose level was $3+$, urinary ketone body level was $3+$, urinary protein level was $2+$, and urinary $\mathrm{pH}$ level was $5.0(6.0-7.0)$.

After admission, she underwent oxygen inhalation, mediastinal gas drainage through the neck, massive fluid replacement, and correction of acidosis, regulating her blood

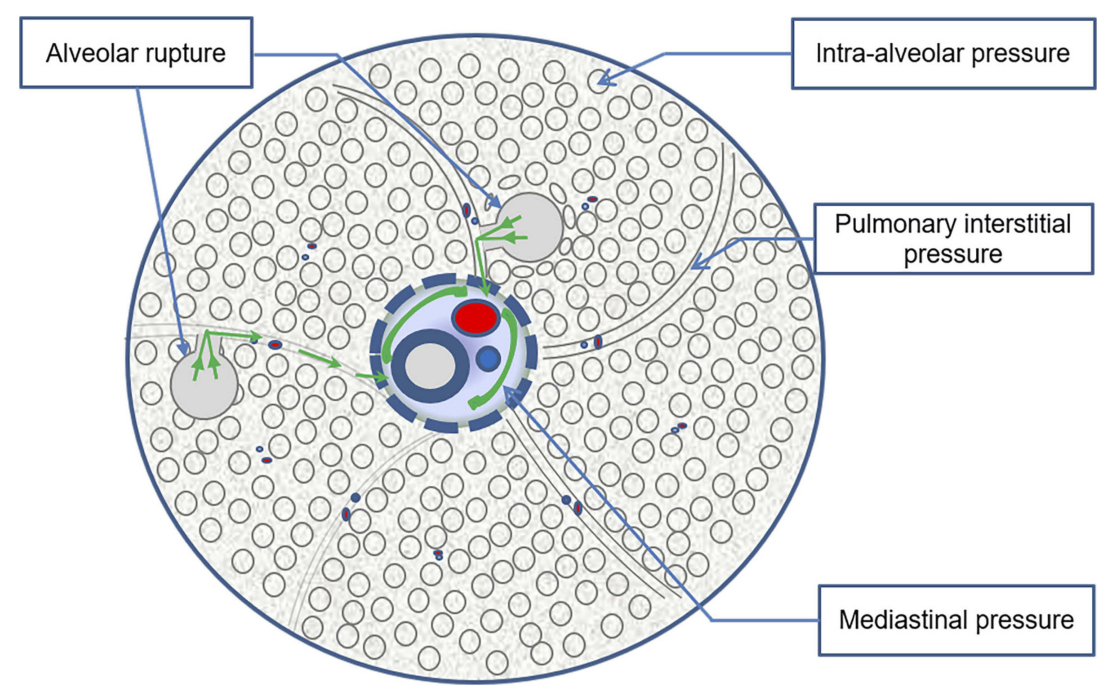

Figure I Pathogenesis of DKA complicated with PM. Under normal physiological conditions, the intra-alveolar pressure > pulmonary interstitial pressure > mediastinal pressure. Kussmaul breathing and severe vomiting caused a sudden increase in intra-alveolar pressure, which caused alveolar rupture and gas from the ruptured alveoli to flow to the pulmonary interstitial region and the mediastinum, resulting in mediastinal emphysema. The Kussmaul breathing and severe vomiting symptoms caused by diabetic ketoacidosis aggravated the changes in the alveolar pressure gradient, making it more likely that pneumomediastinum formed. 
glucose level and other symptomatic support treatment was provided. The symptoms of nausea and vomiting were gradually relieved, the breathing gradually became stable, and the heart rhythm dropped to the normal range after $24 \mathrm{hrs}$. Three days later, when the patient's condition was stable, the oesophageal barium meal examination excluded oesophageal rupture. The accumulation of gas in the mediastinum of the patient evident in the chest CT had completely disappeared after 10 days. Standard hypoglycaemic therapy was continued after discharge. To date, which is after approximately 2 years, the patient has not exhibited recurrence.

\section{Literature Review and Summary}

With "pneumomediastinum, diabetic ketoacidosis" as keywords, 79 patients with diabetic ketoacidosis associated with pneumomediastinum were identified in the CNKI (China National Knowledge Infrastructure), Wanfang, Google Scholar and PubMed databases. The characteristics of the 79 patients are shown in Table 1.

\section{Discussion}

There are many causes of pneumomediastinum in clinical practice, such as chest trauma, oesophageal perforation, iatrogenic injury (tracheotomy, artificial pneumothorax pneumoperitoneum, bronchoscopy, esophagoscopy, tooth extraction, etc.). Childbirth, diving, drug abuse, cough, and asthma are unusual, and pneumomediastinum caused by diabetic ketoacidosis is very rare. ${ }^{2,6-8}$

Because it is extremely rare, clinicians often fail to make a definite diagnosis early. If patients cannot be diagnosed and treated in a timely manner, malignant events can occur in patients. Diabetic ketoacidosis with pneumomediastinum is often associated with pneumopericardium and subcutaneous emphysema, occasionally epidural pneumatosis, ${ }^{1,3,9-11}$ and even pneumothorax ${ }^{3,12,13}$ and stress-induced cardiomyopathy. ${ }^{14}$ Therefore, it is of great importance to thoroughly understand the pathogenesis, clinical symptoms, diagnostic methods and treatment of pneumomediastinum caused by diabetic ketoacidosis.

\section{Pathogenesis}

Most scholars ${ }^{1,3,15}$ believe that the cause of mediastinal gas accumulation in patients with diabetic ketoacidosis complicated with pneumomediastinum is gas entering the pulmonary interstitium due to various causes of alveolar rupture. The bronchus and blood vessels located around the hilum of the lung in the mediastinum are surrounded by interstitial sheaths containing gaps that extend along the segmental bronchi, sub-
Table I Clinical Data of 79 Cases with DKA Complicated with PM

\begin{tabular}{|c|c|c|c|}
\hline & Mean & S.D & $\begin{array}{l}\text { Number of } \\
\text { Patients (n) }\end{array}$ \\
\hline Age(years) & 21 & 9.1 & 79 \\
\hline Sex & & & \\
\hline Male(n) & & & 54 \\
\hline Female(n) & & & 25 \\
\hline Blood glucose(mmol/L) & 36.2 & 17.2 & 66 \\
\hline Heart rate(bmp) & 127 & 17.9 & 46 \\
\hline Respiratory rate(Times/min) & 33 & 8.3 & 39 \\
\hline $\mathrm{PH}$ & 7.08 & 0.2 & 53 \\
\hline $\mathrm{PaCO}_{2}(\mathrm{mmHg})$ & 19.3 & 13.9 & 34 \\
\hline $\mathrm{HCO}^{-}(\mathrm{mmHg})$ & 7.6 & 4.3 & 44 \\
\hline Blood pressure systolic $(\mathrm{mmHg})$ & 122 & 24.9 & 44 \\
\hline Blood pressure diastolic $(\mathrm{mmHg})$ & 72 & 17.0 & 44 \\
\hline Serum sodium(mmol/L) & 131.8 & 9.9 & 28 \\
\hline Serum potassium(mmol/L) & 4.9 & 1.4 & 29 \\
\hline White blood cell count $\left(\times 10^{9} / \mathrm{L}\right)$ & 24.18 & 9.5 & 36 \\
\hline History of diabetes(n) & & & 49 \\
\hline Vomiting(n) & & & 56 \\
\hline Esophagography or gastroscopy(n) & & & 21 \\
\hline $\begin{array}{l}\text { Complicated with epidural } \\
\text { pneumatosis ( } n \text { ) }\end{array}$ & & & 5 \\
\hline Complicated with pneumothorax (n) & & & 3 \\
\hline
\end{tabular}

bronchus, and parallel vessels to the lung parenchyma, and the pressure in the mediastinum is generally less than that in the alveoli. This pressure difference causes the pulmonary interstitial gas to flow backwards into the mediastinal space after alveolar rupture, leading to pneumomediastinum. This gas flow was described in detail by the Macklin ${ }^{16}$ brothers as early as 1944, and they pointed out that after the alveolar gas flows backwards into the mediastinum, with an increase in the gas and pressure in the mediastinum, it enters the pericardium and epidural, retroperitoneal, and subcutaneous spaces and even breaks through the mediastinal pleura to form pneumothorax, this mechanism is called the Macklin effect by some scholars. ${ }^{17}$ Why does diabetic ketoacidosis cause alveolar rupture? Based on the previous literature, ${ }^{1-4,17,18}$ it was found that the cause of alveolar rupture is a change in the pressure difference between internal and external alveoli, and 
most of the patients with acidosis also had Kussmaul breathing (deep and laboured breathing) and severe vomiting. There are root causes of pneumomediastinum in patients with diabetic ketoacidosis. Bullaboy reported that Kussmaul respiration can increase the alveolar pressure to $20-30 \mathrm{mmHg}$, which is sufficient to cause alveolar rupture. ${ }^{18}$ Severe vomiting could lead to changes in the alveolar pressure gradient in the chest. Mediastinal air accumulation caused by tracheal rupture is rare because severe vomiting is caused by diabetic ketoacidosis. However, it is not clear whether mediastinal air is caused by tracheal rupture or alveolar rupture. ${ }^{19}$ According to the previous literature, almost all patients had Kussmaul respiratory disease, approximately $70.9 \%$ of the patients (56 patients) had definite vomiting symptoms before chest discomfort developed, and almost all of them had no history of complicated lung diseases. The average respiratory rate of the patients was approximately 33 times per minute. That is why diabetic ketoacidosis patients associated with pneumomediastinum. It is interesting to note that the average age of all the patients was 21 years, and $68.4 \%$ of the patients were male. This result may be due to the stronger muscle strength of the ventilator in young men. Deep rapid breathing and severe vomiting are more likely to cause greater alveolar pressure differences, so it is more likely to cause pneumomediastinum, which confirms the above mechanism (Figure 1).

\section{Clinical Symptoms and Signs}

Kussmaul breathing, severe vomiting and chest pain are common symptoms in patients with diabetic ketoacidosis associated with pneumomediastinum. Some patients have complications such as dysphagia, dysphonia, somnolence and so on. Excessive accumulation of polyketone bodies and acidic substances in patients with diabetic ketoacidosis causes deep and rapid breathing, and the patient can develop a mental disorder after a long period of time without remission. Some patients develop severe vomiting due to an electrolyte imbalance, and increased secretion of prostacyclin in the body stimulates the gastrointestinal mucosa. ${ }^{20}$

Retrosternal pain is a concomitant symptom in almost all patients, mainly due to the traction and displacement of mediastinal tissue by gas in the mediastinum, which is often located behind the sternum. It increases with activity and breathing. Pain can radiate to the neck, back, shoulders, arms and even the abdomen. When the mediastinal gas enters the posterior pharyngeal wall or the larynx, a small number of patients may have difficulty swallowing and pronouncing words. When air gathered in the mediastinal space enters the retroperitoneum, abdominal discomfort may also develop in some patients. $^{21}$

The common signs of diabetic ketoacidosis associated with pneumomediastinum are crepitus in the anterior cervical region, deep and laboured breathing (Kussmaul breathing), an increased ventricular heart rate, a positive Hamman sign, and the smell of rotten apples when breathing. In most patients, the blood pressure is normal, bilateral lung auscultation is generally not positive (unless complicated with pneumothorax), the heart rhythm is regular, and the oxygen saturation is normal. As mentioned above, excessive mediastinal gas can cause subcutaneous emphysema in the anterior neck and chest wall, and ketoacidosis can lead to deep and laboured breathing, an accelerated heart rate and a rotten apple smell when breathing. Hamman's crunch is a typical sign of pneumomediastinum, and it is synchronous with cardiac systole and is composed of crepitus and popping noises that are the louder where is located at the apex of the heart, the lower end of the sternum or the left edge of the sternum. ${ }^{5}$ This is especially true in the inspiratory and left recumbent position. The cause of Hamman's crunch is retrograde flow of the gas accumulated from the vascular sheath after alveolar rupture into the pericardial cavity, resulting in pericardial gas accumulation. Severe pericardial gas accumulation leads to pericardial tamponade, resulting in circulatory disturbance and failure, which can lead to the death of patients with diabetic ketoacidosis with pneumomediastinum.

\section{Diagnostic Methods}

Most diabetic ketoacidosis patients with pneumomediastinum were admitted to the hospital because of Kussmaul respiration, severe vomiting and chest pain. Chest X-ray or chest CT scans can reveal mediastinal air accumulation. For patients who additionally have subcutaneous gas, a chest X-ray can be used to easily detect pneumomediastinum, but for patients with a small amount of mediastinal air, chest CT scans are more accurate. It is also easier to detect pneumopericardium and epidural pneumatosis with chest CT scans. In some patients, chest CT examinations revealed scattered air shadows in the bilateral pulmonary stroma, intravascular sheath, bronchial sheath and mediastinum (Figure 2), which can be used to determine whether the cause of mediastinal gas is rupture of the oesophagus or trachea. Gas accumulation due to oesophageal and tracheal rupture is limited to the periphery of the rupture and causes soft tissue swelling and even local gasliquid level performance. Chest CT scans have a more intuitive performance in determining the degree of 

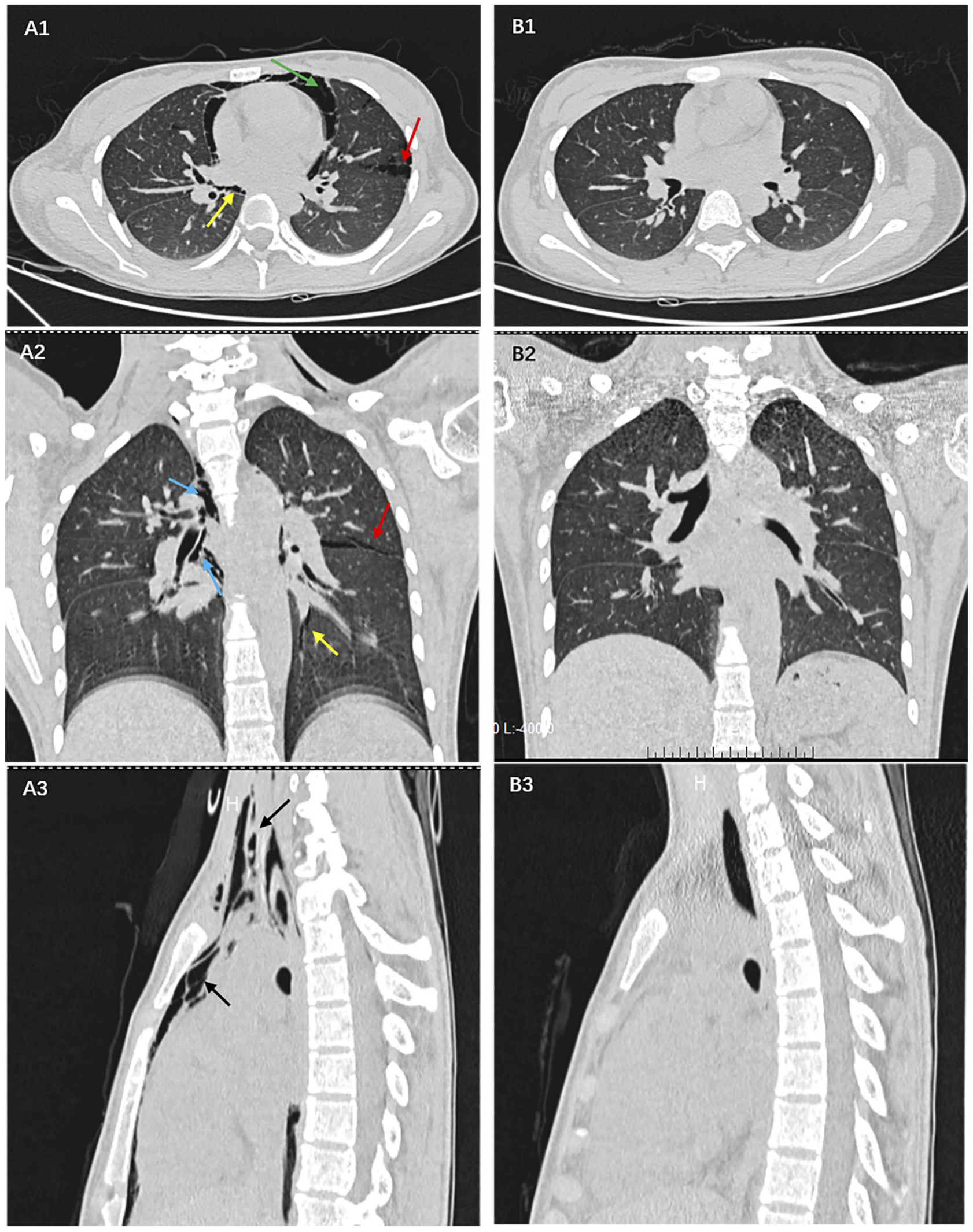

Figure 2 In (AI-3) a large amount of gas has collected in the pericardium (green arrow), in the interstitial lung (red arrow), in the bronchial sheath (blue arrow), and in the pulmonary vascular sheath (yellow arrow), before entering the mediastinum (black arrow), which is a unique manifestation of diabetic ketoacidosis with pneumomediastinum. (BI-3) shows that there is no accumulation of gas in the pericardium, interstitial lung, bronchial and pulmonary vascular sheath, and mediastinum after correction for acidosis and hyperglycemia. 
pericardial tamponade, so these scans provide a basis for a timelier detection and treatment of serious cases of pericardial tamponade. Therefore, all patients suspected of having pneumomediastinum should be examined by chest CT scans because chest CT scan also allow clinicians to better evaluate the progress and remission of the lesion.

Arterial blood gas, blood glucose and urinary ketone body analyses are used for the diagnosis of diabetic ketoacidosis. The arterial $\mathrm{pH}$ values of diabetic ketoacidosis patients with pneumomediastinum were low, with an average of 7.08 , because severe acidosis can aggravate the symptoms of Kussmaul respiration and severe vomiting, resulting in alveolar rupture and mediastinal gas accumulation. According to a review of the literature, the average number of white blood cells for these patients were $24.2 \times$ $10^{9} \mathrm{~L}$, but a careful analysis of the imaging data showed that most of the patients had no imaging manifestations of infection in the lungs. Therefore, the authors believe that the higher white blood cell count found in these patients is not related to pulmonary infection but is mainly related to the stress response of acidosis and may also be related to mediastinal infection caused by the accumulation of gas in the mediastinum.

The symptoms of pneumomediastinum caused by esophageal rupture are similar to those of diabetic ketoacidosis with pneumomediastinum, and esophageal rupture is harmful, with a mortality rate of $40 \%$; some cases can require emergency surgical treatment. ${ }^{9}$ Therefore, the exclusion of esophageal rupture is the primary task in the diagnosis of diabetic ketoacidosis complicated with pneumomediastinum.

According to the literature analysis, $, 1,3,10,11,13,22-3621$ patients with diabetic ketoacidosis complicated with pneumomediastinum underwent esophagography or gastroscopy. Only one patient was found to have a tear in the cardia. (The authors considered that it might be caused by vomiting. However, it was not the cause of pneumomediastinum). ${ }^{25}$ The remaining patients did not have esophageal rupture.

One of the patients was admitted to the hospital because of a coma complicated with pneumomediastinum. Because esophageal rupture could not be ruled out at the time, thoracotomy was performed, and esophageal rupture was not found. After the symptomatic treatment of ketoacidosis, the mediastinal gas disappeared. However, hyperglycaemia was found in this patient upon and after admission, but the attending physician did not pay attention to it. Therefore, the author believes that attending doctors may not fully understand the existence of diabetic ketoacidosis complicated with pneumomediastinum, leading them to open the chest blindly. ${ }^{22}$

Most of the cases of esophageal rupture are triggered by an event, such as trauma, eating sharp and hard foods, and invasive operations, etc., and most of them are accompanied by obvious symptoms of mediastinal infection. Combined with those results in the previous literature,, 32 the authors believe that for patients with diabetic ketoacidosis complicated with pneumomediastinum, if there is no definite cause of esophageal rupture and no obvious signs of mediastinal infection, symptomatic support treatment ketoacidosis should be administered first. Then, esophagography and gastroscopy should be performed to determine whether there is any injury to the esophagus if necessary.

\section{Treatment and Prognosis}

When the diagnosis of diabetic ketoacidosis complicated with pneumomediastinum is clear, these patients are mainly treated for diabetic ketoacidosis with treatments such as fluid replacement, hypoglycaemia, correction of acidosis and preventive anti-infection treatments. However, for some patients with severe subcutaneous pneumatosis and pericardial tamponade, for whom the stability of respiratory circulation is affected, subcutaneous pneumatosis decompression and even pericardial decompression should be performed immediately. For this patient in our hospital, a large amount of pneumopericardium was observed on the chest CT scans, and the heart structure could not be detected with Doppler echocardiography. However, after acidosis treatment, the patient's circulation became stable, and the sound of the heart gradually became clear. The author did not further address the pericardial gas tamponade.

The analysis of the previous literature showed that no patients was given pericardial decompression, but one patient died of cardiac arrest after admission. ${ }^{33}$ It is not known whether the cause of death was related to severe pericardial tamponade. Therefore, the authors believe that the treatment of patients with severe pericardial tamponade should mainly include acidosis treatment; if the symptoms of pericardial tamponade are not relieved after the correction of acidosis or when the accumulation of pericardial gas increases, an additional invasive operation can be carried out to alleviate the symptoms of pericardial tamponade.

The rapid progression of acute ketoacidosis can also lead to stress-induced cardiomyopathy. It is necessary to fully understand the comorbidities and provide symptomatic supportive treatment. If it cannot be identified and treated in a timely manner, a malignant event can easily 
occur. ${ }^{14}$ However, for some patients with epidural pneumatosis, pneumothorax and stress-induced cardiomyopathy, after the correction of acidosis, the symptoms of Kussmaul respiration and vomiting were relieved, and the accumulation of gas in the mediastinum, epidural and subcutaneous regions and even the thoracic cavity was gradually absorbed. Most of the patients were fully cured and discharged from the hospital within 1 to 2 weeks.

Most of the patients with diabetic ketoacidosis complicated with pneumomediastinum had a good prognosis, and the mortality was low. In China, a patient suffered from sudden cardiac arrest and respiratory arrest $10 \mathrm{hrs}$ after admission. She died of aggravation of mediastinal pneumatosis and subcutaneous gas after rescue measures such as tracheal intubation and assisted breathing were administered. ${ }^{33}$ One patient died in the UK due to a failure to control the severe pulmonary infection. ${ }^{34}$ In the previous literature, ${ }^{35}$ only one patient had recurrence twice after the treatment of pneumomediastinum, but the specific reason is not clear. It may be that other patients with diabetic ketoacidosis also develop pneumomediastinum again, but their symptoms are mild, and chest CT scans were not performed, so pneumomediastinum could not be diagnosed. Therefore, it is necessary to review chest CT scans when respiratory and circulatory abnormalities occur in patients with ketoacidosis.

\section{Conclusion}

Diabetic ketoacidosis with pneumomediastinum is a rare disease that is relatively common in young men. The cause of diabetic ketoacidosis with pneumomediastinum is alveolar rupture, which is caused by a change in the alveolar pressure gradient caused by Kussmaul respiration and severe vomiting, which occur due to ketoacidosis. The prognosis is generally good, but it can be fatal when complicated with severe pneumopericardium.

\section{Informed Consent}

Written informed consent had been provided by the patient to have the case details and any accompanying images published. This was an observational case and institutional approval was not required, because all treatment received by this patient is conventional therapy. The other patients' information in this article comes from the references.

\section{Acknowledgments}

The study was supported by a grant from the Henan Medical Science and Technology Research Program (no. 2018020567) as part of the Capital Characteristic Clinical Application Research project.

\section{Disclosure}

The authors declare no conflicts of interest in this work.

\section{References}

1. Pooyan P, Puruckherr M, Summers JA, et al. Pneumomediastinum, pneumopericardium, and epidural pneumatosis in DKA. J Diabetes Complications. 2004;18(4):242-247. doi:10.1016/S1056-8727(03) 00059-X

2. Kouritas VK, Papagiannopoulos K, Lazaridis G, et al. Pneumomediastinum. J Thorac Dis. 2015;7(Suppl 1):S44-49. doi:10.3978/j. issn.2072-1439.2015.01.11

3. Pauw RG, Ts VDW, van Dullemen HM, et al. Mediastinal emphysema complicating diabetic ketoacidosis: plea for conservative diagnostic approach. Neth J Med. 2007;65(10):368-371.

4. Russo A, Del VC, Zaottini A, et al. Role of emergency thoracic ultrasonography in spontaneous pneumomediastinum. Two case report. G Chir. 2012;33(33):285-296.

5. Hamman L. Spontaneus mediastinal emphysema. Bull Johns Hopkins Hosp. 1939;64:1-21.

6. Zylak CM, Standen JR, Barnes GR, et al. Pneumomediastinum revisited. Radiographics. 2000;20(4):1043-1057. doi:10.1148/radiographics.20.4.g00j1131043

7. Garcipérez de Vargas FJ, Gómez Barrado JJ, Luis Moyano Calvente S, et al. Pneumopericardium and pneumomediastinum in a diabetic and cocaine user patient. Endocrinol Nutr. 2013;60(8):e34. doi:10.1016/j.endonu.2012.12.001

8. Potu KC, Gedela M, Shaikh KA, et al. An unusual presentation of an unusual disease: spontaneous pneumomediastinum. $S$ D Med. 2016;69(11):495-497.

9. Pain AR, Pomroy J, Benjamin A. Hamman's syndrome in diabetic ketoacidosis. Endocrinol Diabetes Metab Case Rep. 2017;2017(1). doi:10.1530/EDM-17-0135

10. Ahmed M, Healy ML, O'Shea D, et al. Epidural pneumatosis associated with spontaneous pneumomediastinum: a rare complication of diabetic ketoacidosis. BMJ Case Rep. 2016;2016:bcr2016216295. doi:10.1136/bcr-2016-216295

11. Hall WB, Aris RM. Epidural pneumatosis and diffuse soft tissue free air as a complication of diabetic ketoacidosis. Am J Respir Crit Care Med. 2012;185(4):e5. doi:10.1164/rccm.201105-0916IM

12. Weathers LS, Brooks WG, DeClue TJ. Spontaneous pneumomediastinum in a patient with diabetic ketoacidosis: a potentially hidden complication. South Med J. 1995;88:483-484. doi:10.1097/00007611-19950400000022

13. Gabor SE, Renner H, Maier A, et al. Tension pneumomediastinum after severe vomiting in a 21-year-old female. Eur J Cardiothorac Surg. 2005;28:502-503. doi:10.1016/j.ejcts.2005.05.015

14. Lane AS, Champion B, Orde S, et al. Diabetic ketoacidosis due to fulminant type 1 diabetes: a rare subtype of type 1 diabetes leading to unusual sequelae. J Intensive Care Soc. 2015;16(1):64-70. doi:10.1177/ 1751143714551249

15. Hu Y, Ma Z, Guo Z, et al. Type 1 diabetes mellitus is an independent risk factor for pulmonary fibrosis. Cell Biochem Biophys. 2014;70 (2):1385-1391. doi:10.1007/s12013-014-0068-4

16. Macklin MT, Macklin CC. Malignant interstitial emphysema of the lungs and mediastinum as an important occult complication in many respiratory diseases and other conditions: an interpretation of the clinical literature in the light of laboratory experiment. Medicine. 1944;23(4):281-358. doi:10.1097/00005792-194412000-00001

17. Murayama S, Gibo S. Spontaneous pneumomediastinum and Macklin effect: overview and appearance on computed tomography. World J Radiol. 2014;6(11):850-854. doi:10.4329/wjr.v6.i11.850

18. Bullaboy CA, Jennings RB Jr, Johnson DH, et al. Radiological case of the month. Pneumomediastinum and subcutaneous emphysema caused by diabetic hyperpnea. Am J Dis Child. 1989;143(1):93-94. 
19. Stevens MS, Mullis TC, Carron JD. Spontaneous tracheal rupture caused by vomiting. Am J Otolaryngol. 2010;31(4):276-278. doi:10.1016/j.amjoto.2009.02.008

20. Vermeersch N1, Stolte C, Fostier K, et al. An unusual case of hyperglycemia, abdominal pain, and increased anion gap acidosis. J Emerg Med. 2010;39(4):449-453. doi:10.1016/j.jemermed.2007.11.004

21. Sun D, Chen F, Wang C. An unusual etiology of abdominal pain and spontaneous pneumomediastinum in a young adult. Am J Emerg Med. 2014;32(8):947.e1-2. doi:10.1016/j.ajem.2014.01.035

22. Alkhuja S, Gazizov N, Charles G. Pneumomediastinum complicating diabetic ketoacidosis and Boerhaave's syndrome. Case Rep Med. 2013;2013:598720.

23. Flores López EN, Alvarez Torrecilla LC, López Alférez R. Spontaneous pneumomediastinum in a patient with diabetic ketoacidosis. Arch Bronconeumol. 2014;50(10):459. doi:10.1016/j.arbres.2013.09.004

24. Speksnijder L, Duvekot JJ, Duschek EJ, Jebbink MC, Bremer HA. Spontaneous pneumomediastinum: a rare presentation of diabetic ketoacidosis in a pregnant woman. Obstet Med. 2010;3(4):158-160. doi:10.1258/om.2010.100028

25. Lin SY, Chang CT, Chen CC, et al. Diabetic ketoacidosis complicated by pneumomediastinum and Mallory-Weiss tear in a young boy with new onset Type 2 diabetes. South Med J. 2008;101(7):769-770. doi:10.1097/SMJ.0b013e318178da02

26. Xing XQ, Wang J, Yang ZJ, et al. A case of diabetic ketoacidosis complicated with pneumomediastinum. Chin J Tuberculosis Respir Dis. 2011;11:859-860.

27. Chapdelaine J, Beaunoyer M, Daigneault P, et al. Spontaneous pneumomediastinum: are we overinvestigating? J Pediatr Surg. 2004;39 (5):681-684. doi:10.1016/j.jpedsurg.2004.01.045
28. Panacek EA, Singer AJ, Sherman BW, et al. Spontaneous pneumomediastinum: clinical and natural history. Ann Emerg Med. 1992;21 (10):1222-1227. doi:10.1016/S0196-0644(05)81750-0

29. Monjardino J, Smith D, Tisdall M, et al. Hyperpnoea and emesis in a diabetic man. J R Soc Med. 2002;95(10):502-503. doi:10.1177/ 014107680209501007

30. Levsky JM, Feuer BH, Di Vito J Jr. Pneumomediastinum in a patient with diabetic ketoacidosis. J Emerg Med. 2004;26(2):233-235. doi:10.1016/j.jemermed.2003.09.006

31. Freixinet J, Garcia F, Rodriguez PM, et al. Spontaneous pneumomediastinum long-term follow-up. Respir Med. 2005;99(9):1160-1163. doi:10.1016/j.rmed.2005.02.025

32. Hamamoto H, Sakaguchi K, Muro S, et al. A case of Hamman's syndrome associated with acute-onset type 1 diabetes mellitus presenting with abdominal pain. Nihon Shokakibyo Gakkai Zasshi. 2015;112(5):856-862. doi:10.11405/nisshoshi.112.856

33. Shang J, Yao HB, Zhang YB. Pneumomediastinum in a patient with diabetic ketoacidosis: one case report. Tianjin Med J. 2011;8:691.

34. Ruttley M, Mills RA. Subcutaneous emphysema and pneumomediastinum in diabetic keto-acidosis. Br J Radiol. 1971;44(525):672-674. doi:10.1259/0007-1285-44-525-672

35. Nessan VJ. Recurrent pneumomediastinum in diabetic ketoacidosis. Postgrad Med. 1974;55(6):139-140. doi:10.1080/00325481.1974. 11713791

36. Gong WK, Lai JB, Hu JY, et al. Spontaneous pneumomediastinum in a patient with diabetic ketoacidosis: one case report. Chin $J$ Endocrinol Metab. 2016;9:784-786.

\section{Publish your work in this journal}

Diabetes, Metabolic Syndrome and Obesity: Targets and Therapy is an international, peer-reviewed open-access journal committed to the rapid publication of the latest laboratory and clinical findings in the fields of diabetes, metabolic syndrome and obesity research. Original research, review, case reports, hypothesis formation, expert opinion and commentaries are all considered for publication. The manuscript management system is completely online and includes a very quick and fair peer-review system, which is all easy to use. Visit http://www.dovepress.com/testimonials.php to read real quotes from published authors.

Submit your manuscript here: https://www.dovepress.com/diabetes-metabolic-syndrome-and-obesity-targets-and-therapy-journal 